\title{
EVALUACIÓN DE EVENTOS SECOS Y HÚMEDOS EN EL CONTEXTO DEL CAMBIO CLIMÁTICO: EL CASO DEL SUR DE LA REGIÓN PAMPEANA (ARGENTINA)
}

\author{
Federico Ferrelli, Andrea Soledad Brendel ${ }^{I}$, Piccolo María Cintia ${ }^{l}$, Perillo Gerardo M. \\ Eduardo ${ }^{1}$ \\ Instituto Argentino de Oceanografía
}

\section{RESUMEN}

El objetivo de este estudio fue analizar la duración, intensidad, periodicidad y frecuencia de los eventos secos y húmedos durante i) pasado reciente (1977-1997), ii) presente (1998-2018), iii) futuro cercano (2019-2039) y iv) futuro lejano (2079-2099) en el sur de la Región Pampeana (Argentina). Se aplicó el Índice Estandarizado de Precipitación a escala anual, estacional, semestral y mensual, utilizando datos de doce estaciones meteorológicas. Los resultados fueron interpolados con el método Kriging en un Sistema de Información Geográfica y de esta manera se obtuvo la distribución espacial de los eventos analizados. El área de estudio evidenció una prolongación de los períodos secos, alcanzando 12 meses de duración en el futuro lejano. Los períodos húmedos presentaron un comportamiento similar entre las épocas estudiadas. Teniendo en cuenta su distribución espacial, el sur del área de estudio fue la que registró cambios significativos, dado que presentará mayor frecuencia de eventos secos y húmedos en el futuro. Esta situación podría afectar las actividades económicas que actualmente se desarrollan en el área. La información resultante es esencial para que la población conozca las situaciones a las que podrían estar expuestos ante las fluctuaciones que tendrá la precipitación en situaciones meteorológicas futuras.

Palabras clave: Modelos climáticos; datos in situ; planificación del territorio; región semiárida de Argentina.

\section{ASSESSMENT OF DRY AND WET EVENTS IN THE CONTEXT OF CLIMATE CHANGE: A CASE OF THE SOUTH OF PAMPAS (ARGENTINA)}

\section{ABSTRACT}

This study aims to analyze the duration, intensity, periodicity, and frequency of dry and wet events in the south of Pampas (Argentina), considering i) recent past (1977-1997), ii) present (1998-2018), iii) near future (2019-2039), and iv) far future (2079-2099). In this sense, monthly rainfall data were assessed from twelve weather station using the Standardized Precipitation Index with 1-month, 3-months,6-months and 12-months timescales. Kriging interpolation method was applied to analyze information spatially. The results showed an extension of dry events with a duration up to 12 months in the far future, while wet events showed a similar behavior among the considered periods. It is worth noting that the south part of the study area will present a higher frequency of wet and dry events, which will generate a more variable rainfall regime affecting the economic activities of the area. The information obtained will be essential for decision-makers and agricultural producers because it will allow them to know the rainfall fluctuation in future situations.

Key words: Climate models, in situ data; land management; semiarid region of Argentina.

\footnotetext{
${ }^{1}$ Instituto Argentino de Oceanografía (IADO), Universidad Nacional del Sur (UNS)-CONICET, Bahía Blanca-Argentina. E-mail: fferrelli@criba.edu.ar; sbrendel@iado-conicet.gob.ar; ofpiccolo@criba.edu.ar; gmeperillo@criba.edu.ar
}

Fecha de recepción: 10 de junio de 2020. Fecha de aceptación: 27 de agosto de 2020 


\section{INTRODUCCIÓN}

En el contexto actual del cambio climático, los eventos pluviométricos extremos adquieren relevancia, dado que modifican los patrones climáticos regionales al incrementar su frecuencia e intensidad (PALMER y RAISANEN, 2002; HAYES et al., 2004; NAM et al., 2015). Los períodos secos y húmedos son componentes de la variabilidad pluviométrica y constituyen los fenómenos climáticos de mayor impacto sobre la hidrología y las actividades agrícolas de una región (ALIAGA et al., 2016). Una de las principales dificultades para estudiar estos eventos recae en que no existe una única definición de sequía y eventos húmedos. Los mismos se adaptan a los distintos tipos de clima (WILHITE et al., 2007).

En este contexto, se definieron a las sequías como un evento en el que el régimen de precipitaciones fue inferior al calculado para un período de tiempo mayor a 30 años. Una sequía meteorológica es un período de tiempo (establecido en meses o años), en donde el aporte de humedad es inferior al climáticamente esperado. Su ocurrencia depende de la evapotranspiración, el déficit de las precipitaciones y del condicionamiento impuesto por los cambios en la presión causantes de las alteraciones en la circulación general de la atmósfera. En la Argentina, se asocia a condiciones predominantemente anticiclónicas y a cambios en la cobertura nubosa que persisten durante un cierto período de tiempo. En contraposición, los eventos húmedos se determinan cuando se generan las condiciones opuestas (VALIENTE, 2001).

Las sequías ocurren en todo el mundo, aún en regiones húmedas (CHEN et al., 2013). La ocurrencia de estos eventos están estrechamente ligadas con procesos erosión y desertificación sobre áreas extensas de nuestro planeta (TSESMELIS et al., 2019).Las tendencias actuales indican que, en el futuro, los eventos pluviométricos serán más severos (MUKHERJEE et al., 2018). Existen regiones del mundo donde las precipitaciones se incrementarán y, por lo tanto, las inundaciones serán más abundantes, mientras que, en otras, las sequías tendrán mayor duración e intensidad (CHEN et al., 2013; FERRELLI et al., 2019). La variabilidad pluviométrica genera consecuencias en la economía regional, a partir del impacto socioeconómico que causan (BRENDEL et al., 2017). Entre los efectos sobre la población, se destacan la accesibilidad al agua potable, la provisión de energía, el acceso a plantas medicinales, el desplazamiento o desaparición de animales y recursos pesqueros y el aumento de los riesgos de inundación o sequía como consecuencia de los cambios geomorfológicos en las zonas aluviales (HOUGHTON, 1993; OLLERO OJEDA et al., 2006; FERRELLI, 2017).

Las sequías e inundaciones son fenómenos espacio-temporales muy dinámicos, lo que genera que la cuantificación objetiva de su duración, frecuencia, periodicidad e intensidad resulte compleja (DAI, 2011). Al no existir una única definición de sequías, se han diseñado e implementado un amplio número de índices para su evaluación y estudio con el objetivo de mantener un monitoreo constante. En general, estos indicadores utilizan información meteorológica e hidrológica (KEYANTASH y DRACUP, 2002; SHAHID y BEHRAWAN, 2008; WANG et al., 2010; OTKIN et al., 2015, entre otros). Dentro de los métodos desarrollados, el más aplicado en los últimos años ha sido el Índice Estandarizado de Precipitación (IEP) (MC KEE et al., 1993). En comparación con otros índices, como por ejemplo el índice de sequía de Palmer, el IEP es fácil de calcular dado que solo requiere de datos de precipitación. Por lo tanto, es adecuado para el monitoreo de las sequías meteorológicas y los eventos húmedos, permitiendo realizar comparaciones a escala global (YUAN y ZHOU, 2004; DU et al., 2012).

El IEP ha sido utilizado para caracterizar condiciones secas y húmedas en numerosos países y regiones del mundo. Por ejemplo, en Estados Unidos (WU et al. 2007), Brasil (SOBRAL et al., 2019), China (DU et al., 2012) y Argentina (COLLAZO et al., 2019). Además, su aplicación favorece el estudio de la severidad de los eventos secos y húmedos en escenario futuros bajo distintas condiciones de concentración de dióxido de carbono (TSESMELIS et al., 2019).

El estudio de las situaciones pluviométricas futuras es posible debido a la existencia de modelos climáticos globales que caracterizan la precipitación bajo dos escenarios de diferente concentración de dióxido de carbono en la atmósfera. Existen numerosos estudios en los que se han aplicado y se han evidenciado cambios significativos en los patrones de los eventos secos y húmedos (DUBROVSKY et al., 2014; TOUMA et al., 2015). Dentro de este tipo de estudios prevalecen aquellos destinados a identificar la frecuencia, duración, intensidad y extensión de las 
sequías (p.ej., SPINONI et al., 2014). En este contexto, es importante realizar investigaciones que permitan predecir el efecto del cambio climático en la ocurrencia de eventos secos y húmedos e identificar su patrón espacio-temporal para poder diseñar y enmarcar políticas destinadas a la prevención de los impactos negativos de estos eventos (NAM et al., 2015).

En la Región Pampeana (Argentina), el modelo CCSM4 ha sido utilizado con diversos fines, dado su excelente ajuste. Se ha aplicado para cuantificar el impacto del cambio climático sobre los rendimientos de los principales cultivos (ROLLA et al., 2018) y la producción ganadera bovina (ROLLA et al., 2019), evaluar la variabilidad espacio-temporal de la precipitación (MAENZA et al., 2017) y para calcular el IEP y analizar la distribución espacio temporal de las sequías (PENALBA y RIVERA, 2016).

Las regiones semiáridas están expuestas a situaciones de aridez que incrementan su exposición a los procesos de degradación ambiental, por lo que su monitoreo actual y futuro es esencial para el desarrollo de planes de manejo del territorio destinados a mejorar la calidad de vida de la población y el ambiente (BRENDEL et al., 2017; FERRELLI et al., 2019). El área de estudio de esta investigación fue el sudoeste de la provincia de Buenos Aires, Argentina (Figura 1). Está localizado en el extremo sur de la Región Pampeana (Argentina) y se caracteriza por estar enmarcada dentro de los climas semiáridos (ALIAGA et al., 2017). Los eventos extremos pluviométricos son los más intensos de toda la Región Pampeana (ALIAGA et al., 2016). Además, presenta una marcada estacionalidad térmica que permite la diferenciación de veranos e inviernos. Su régimen pluviométrico oscila desde más de $800 \mathrm{~mm}$ al noreste y disminuye en sentido noreste-sudoeste alcanzando $400 \mathrm{~mm}$. Esto marca la presencia de regímenes húmedos, subhúmedos, áridos y semiáridos en la región, estableciendo una heterogeneidad espacial de las precipitaciones (Figura 1). Los suelos favorecen el desarrollo de las actividades agrícolas y ganaderas, aunque esta aptitud decrece con el gradiente de isohietas. El trigo es el principal cultivo de cosecha y aporta aproximadamente el $12 \%$ de la producción a escala nacional. Este se desarrolla de forma extensiva bajo condiciones de secano lo que determina que el área es altamente dependiente de las precipitaciones (BRENDEL et al., 2017; FERRELLI, 2019).

En la actualidad, no se ha realizado un estudio integral que compare las situaciones pasadas, presentes y futuras de los eventos secos y húmedos, como así también su duración, intensidad, frecuencia y periodicidad. El mismo permitiría inferir los efectos de las variaciones pluviométricas sobre la agricultura de secano, los impactos meteorológicos y aquellos esperables sobre los cuerpos de agua y las aguas subterráneas (TSESMELIS et al., 2019). Las investigaciones enfocados a conocer las características futuras de los eventos pluviométricos extremos son esenciales para el desarrollo sustentable, dado que favorecen la generación anticipada de medidas de adaptación y mitigación (WILHITE et al., 2014; NAM et al., 2015). Por ello y considerando que en los últimos años el cambio climático ha aumentado la frecuencia, severidad, intensidad y frecuencia de las sequías y las inundaciones (SHEFFIELD et al., 2012), el objetivo de este estudio fue evaluar los eventos secos y húmedos en cuatro períodos definidos como pasado reciente, presente, futuro cercano y futuro lejano. La información generada permitirá a los tomadores de decisiones y productores agropecuarios disponer de información útil para conocer las situaciones a las que podrían estar expuestos en situaciones meteorológicas futuras. Además, generarán una base de datos indispensable para orientar, por un lado, las políticas públicas de manejo del territorio y por el otro, para tomar medidas de mitigación, teniendo en cuenta los efectos adversos que los eventos pluviométricos extremos tienen sobre las actividades económicas de las regiones semiáridas. 
FIGURA 1

Localización del área de estudio y de las estaciones meteorológicas utilizadas.

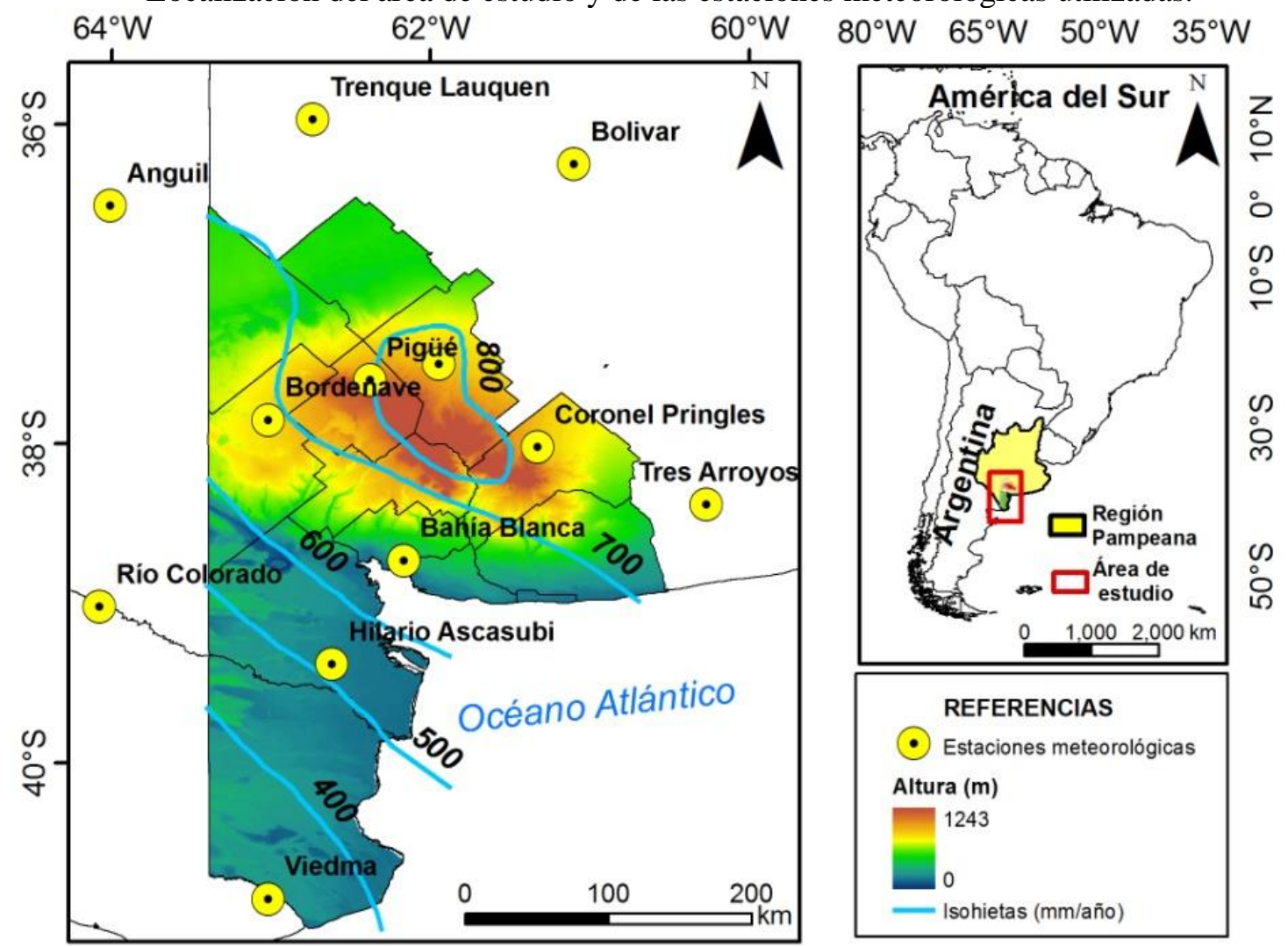

\section{METODOLOGÍA}

El método de trabajo se subdividió en cuatro etapas relacionadas entre sí. En una primera instancia, se estudiaron datos de precipitación registrados en estaciones meteorológicas distribuidas en el área de estudio. Luego, se analizaron series de tiempo provenientes del modelo CCSM4 (Community Climate System Model 4) y se determinaron seis períodos de estudio: pasado reciente, presente, futuro cercano y futuro lejano. Los dos últimos, bajo dos escenarios de concentración de gases de efecto invernadero. Posteriormente, se evaluaron los eventos secos y húmedos a partir del cálculo del IEP en distintas escalas temporales. Finalmente, los resultados fueron representados espacialmente con un Sistema de Información Geográfica.

\subsection{RECOLECCIÓN DE DATOS}

Se utilizaron datos mensuales de precipitación de doce estaciones meteorológicas pertenecientes al Servicio Meteorológico Nacional (SMN, Argentina) y al Instituto Nacional de Tecnología Agropecuaria (INTA, Argentina) localizadas en el área de estudio durante el período 1960-2018 (Figura 1). La homogeneidad y el control de calidad de la información climática se llevó a cabo teniendo en cuenta lo sugerido por FERRELLI et al. (2019) con el objetivo de eliminar los valores atípicos y completar los datos faltantes.

Las series de precipitación del modelo global CCSM4 perteneciente al Centro Nacional de Estudios de la Atmósfera de Estados Unidos (NCAR) fueron adquiridos del Centro de Investigaciones del Mar y la Atmósfera de Argentina (CIMA- http://3cn.cima.fcen.uba.ar). El CIMA analizó un total de 24 modelos y el utilizado en este trabajo es el que mejores resultados proporciona en el área de estudio. Según este organismo, el índice de validación oscila entre 0 y 1 , donde los valores cercanos a 1 son los que representan de manera precisa la información climática futura. En este caso, el modelo CCSM4 presentó un índice de validación de 0,91. Las 
series de tiempo obtenidas de este modelo, se descargaron considerando dos escenarios de concentración de gases de efecto invernadero (RCP - Representative Concentration Pathways, por sus siglas en inglés). Por un lado, el RCP 4.5 hace referencia a un escenario en donde el forzamiento radiativo se estabiliza en $4.5 \mathrm{~W} / \mathrm{m}^{2}$, mientras que el RCP 8.5 indica un escenario en donde las emisiones son elevadas, arrojando un forzamiento radiativo de $8.5 \mathrm{~W} / \mathrm{m}^{2}$.

\subsection{ANÁLISIS DE LA INFORMACIÓN CLIMÁTICA}

Se aplicó una corrección Bias entre los datos registrados in situ con aquellos obtenidos del modelo CCSM4 para el período 1960-2010. Esta corrección es fundamental para determinar las diferencias entre las series modeladas con las observadas y ha sido ampliamente utilizada en trabajos climáticos que utilizan estas fuentes de información (BAIMOUNG et al., 2014; MBAYE et al., 2016; WANG et al., 2016).

Posteriormente, se subdividieron las series de tiempo en períodos con la misma cantidad de años para poder compararlas entre sí. Se consideraron cuatro períodos: i) pasado reciente (19771997), ii) presente (1998-2018), iii) futuro cercano (2019-2039) y iv) futuro lejano (2079-2099). Los dos últimos, se estudiaron bajo dos escenarios de RCP (4.5 y 8.5, respectivamente), contabilizando un total de seis series de tiempo diferentes.

\subsection{EVALUACIÓN DE EVENTOS SECOS Y HÚMEDOS}

Se calculó el Índice Estandarizado de Precipitación (IEP) para caracterizar los eventos secos y húmedos en los períodos de estudio previamente definidos. El mismo fue aplicado teniendo en cuenta las categorías definidas en la Tabla 1 (MC KEE et al., 1993). Este método es el que recomienda la Lincoln Declaration on Drought Indices (HAYES et al., 2011) por ser el más adecuado para estudiar las sequías en América del Sur (PENALBA y RIVERA, 2016). El IEP ha sido ampliamente utilizado para el análisis de las sequías y la identificación de escenarios futuros en distintas escalas espacio-temporales (AL-QUINNA et al., 2011; HEINRICH y GOBIET, 2012; ORLOWSKY y SENEVIRATNE, 2013). Además, es útil para estudiar las actividades que dependen directamente de la precipitación como la agricultura de secano y para evaluar la disponibilidad de agua superficial (STRZEPEK et al., 2010). Por otro lado, facilita la caracterización de los períodos húmedos lo que lo hace un buen indicador para evaluar los riesgos de inundación en una región (MC KEE et al., 1993; EDWARDS et al., 1997; ALIAGA et al., 2017).

TABLA 1

Caracterización de los eventos pluviométricos según el IEP (Mc Kee et al., 1993)

\begin{tabular}{|c|c|}
\hline Categorías del IEP & Valor \\
\hline Extremadamente seco & $<-2$ \\
\hline Muy Seco & $>-1,5<-2$ \\
\hline Moderadamente Seco & $>-1<-1,5$ \\
\hline Normal & $<1>-1$ \\
\hline Moderadamente Húmedo & $>1<1,5$ \\
\hline Muy Húmedo & $>1,5<2$ \\
\hline Extremadamente Húmedo & $>2$ \\
\hline
\end{tabular}

Para calcularlo, se utilizaron las series mensuales de precipitación acumulada. El IEP fue aplicado en distintas escalas temporales: IEP-1 (mensual), IEP-3 (estacional), IEP-6 (semestral) e IEP-12 (anual), caracterizando los períodos según el criterio presentado en la Tabla 1. Esta forma de aplicarlo permite inferir los impactos meteorológicos, sobre la agricultura y la hidrología permitiendo, además, representar los eventos de corta y larga duración. Tras el análisis de las series de tiempo del IEP a distintas escalas temporales, se estudió su frecuencia (identificada por el número de eventos), periodicidad (tiempo transcurrido entre un evento y la ocurrencia de otro), 
duración (extensión temporal en meses de un evento) e intensidad (valor medio del IEP) durante los seis períodos analizados.

\subsection{ANÁLISIS ESPACIAL DE LA INFORMACIÓN}

Teniendo en cuenta que el método de interpolación Kriging es el más utilizado para el análisis de las variables climáticas a escala mundial, se procedió a verificar si éste también lo es en el área de estudio según los siguientes criterios: i) que los datos analizados tengan una distribución normal, ii) que presentan estacionalidad y iii) que no tengan una tendencia lineal (AALTO et al., 2013). Este método de interpolación es de inferencia espacial, es decir, que permite estimar los valores de una variable en lugares no muestreados, utilizando los valores que nos proporciona, en este caso, las estaciones meteorológicas. Por lo tanto, se puede obtener un estimador lineal no sesgado con una varianza mínima. Para realizar estos análisis se utilizó el Software SPSS 20.

Se obtuvo que el método más adecuado para analizar la precipitación en el área de estudio fue el Kriging Universal, dado que este puede utilizarse si se demuestra la existencia de tendencia (UYAN Y KAY, 2010). El mismo asume que la distancia entre los sitios de medición (en este caso, las estaciones meteorológicas) tiene una correlación espacial. Además, su objetivo es minimizar la varianza entre los puntos y producir una mejor estimación lineal no sesgada para una ubicación desconocida (MENAFOGLIO et al., 2013). Como resultado de su aplicación, se generaron productos cartográficos que representaron la periodicidad, la frecuencia, duración e intensidad de los eventos secos y húmedos en el sur de la Región Pampeana para cada uno de los seis períodos definidos previamente. En los mismos, se consideró la misma escala espacial y colorimétrica y se identificaron las áreas más expuestas a sufrir cambios en los distintos escenarios analizados.

\section{RESULTADOS}

Los resultados obtenidos, se dividieron en eventos secos y húmedos. De esta manera, se analizaron los efectos y los cambios espacio-temporales que los mismos tuvieron a lo largo de los seis períodos considerados.

\subsection{EVENTOS SECOS}

Los eventos secos mostraron una intensidad con una tendencia decreciente hacia el futuro. Los más intensos ocurrieron en el pasado reciente (1977-1997), con un valor máximo de IEP de -2,14. Durante 1977-1997, la intensidad de estos marcó una franja de eventos muy secos (IEP >-1,5<2) y extremadamente secos (IEP <-2) en el oeste y norte del área de estudio. En los períodos posteriores, la intensidad de los períodos secos fue disminuyendo, siendo los muy secos los más intensos (Figura 2). Por otra parte, el centro y sur se caracterizó por eventos moderadamente secos (IEP >-1<-1,5). El presente (1998-2018) y futuro cercano (2019-2039) bajo condiciones de RCP 8.5 presentaron una distribución espacial similar. Los eventos secos se localizaron en el oeste y los muy secos al sur. En el futuro lejano (2079-2099) RCP 4.5 solo se observaron períodos muy secos, donde los más intensos se identificaron en el norte y oeste del área de estudio (IEP $=-1,77$ ). Finalmente, en el futuro lejano la intensidad de los eventos secos disminuyó de norte a sur (Figura 2).

La duración (en meses) de los eventos secos mostró marcadas diferencias espaciales. Su principal modificación se produjo en el futuro lejano bajo el escenario RCP 8.5, dado que la mayor parte del área de estudio presentó eventos de mayor duración. Mientras que en el pasado (19771997) la duración máxima fue 13 meses en el sur del área de estudio, la mínima se ubicó al centro, contabilizando un total de 6 meses. En el presente (1998-2018), los eventos secos tuvieron una duración de 10 meses en el norte del área de estudio y disminuyeron a 5 meses en el centro y oeste (Figura 3). Los eventos más extensos fueron de 11 y 10 meses, respectivamente y se localizaron en el oeste y sudoeste del área de estudio, mientras que los mínimos en el norte y el oeste, con un total de 7 y 6 meses de duración, respectivamente (Figura 3). Hacia el futuro lejano, en ambos 
$\mathrm{RCP}$, los eventos secos evidenciaron una duración máxima de 16 meses en el noreste del área de estudio y valores mínimos en el centro (4 meses) (Figura 3).

\section{FIGURA 2}

Distribución espacial de la intensidad de los eventos secos en A. Pasado reciente, B. Presente, C. Futuro cercano RCP 4.5, D. Futuro cercano RCP 8.5, E. Futuro lejano RCP 4.5 y F. Futuro lejano RCP 8.5

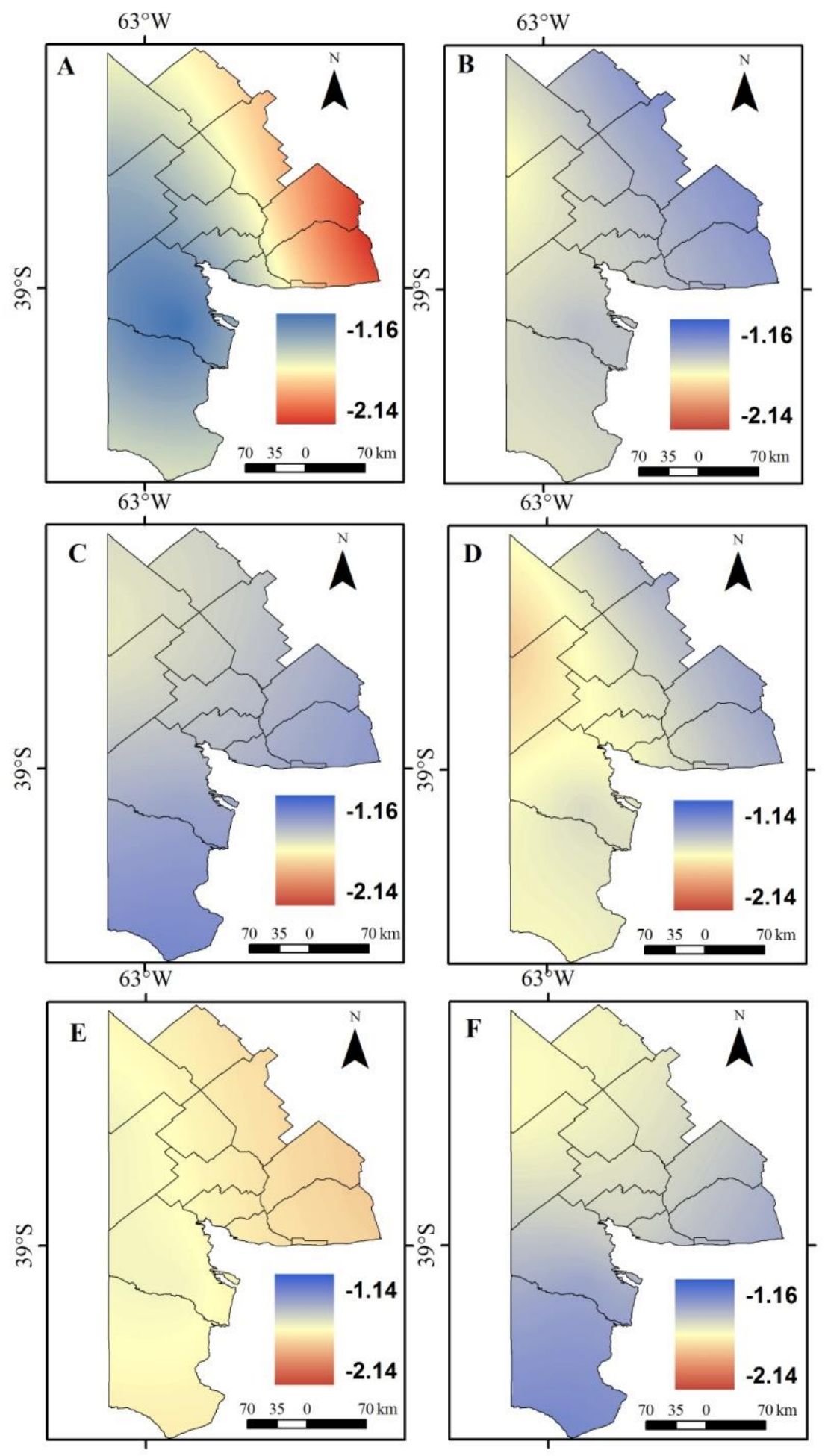


FIGURA 3

Distribución espacial de la duración (meses) de los eventos secos en A. Pasado reciente, B. Presente, C. Futuro cercano RCP 4.5, D. Futuro cercano RCP 8.5, E. Futuro lejano RCP 4.5 y F. Futuro lejano RCP 8.5
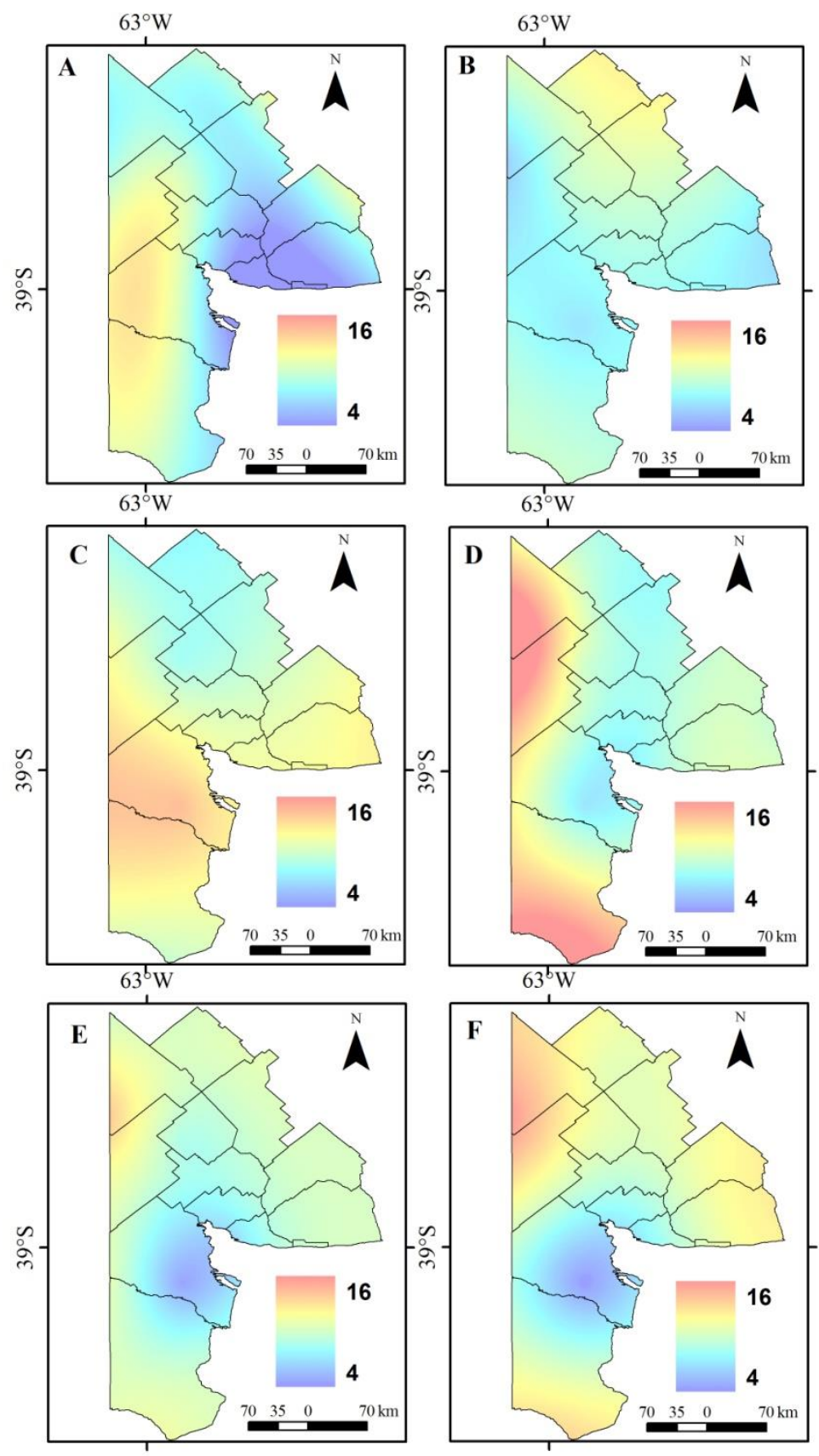

El número de eventos secos fue distinto entre el período pasado y el presente, pero exhibió un patrón similar entre el futuro cercano bajo ambos RCP y entre el período futuro lejano (Figura 4). En el pasado reciente, la zona central y el extremo sur del área de estudio mostraron el mayor número de eventos secos, mientras que el mínimo se observó en el norte y oeste. En el presente, la situación fue la opuesta, dado que se registraron siete eventos secos en el oeste y el noreste y sólo dos en el resto del área de estudio (Figura 4). En el futuro cercano, bajo ambos escenarios RCP, el mayor número de eventos secos se localizó en el sur del área (11 eventos bajo el RCP 4.5 y 10 eventos bajo el RCP 8.5), mientras que en el resto, se registraron los mínimos (5 y 3, respectivamente). Una situación similar (12 eventos) se observó hacia el futuro lejano, en ambos escenarios, dado que la zona de mayor número de eventos secos fue la misma (Figura 4). 
FIGURA 4

Distribución espacial de la frecuencia de los eventos secos en A. Pasado reciente, B. Presente, C. Futuro cercano RCP 4.5, D. Futuro cercano RCP 8.5, E. Futuro lejano RCP 4.5 y F. Futuro lejano RCP 8.5
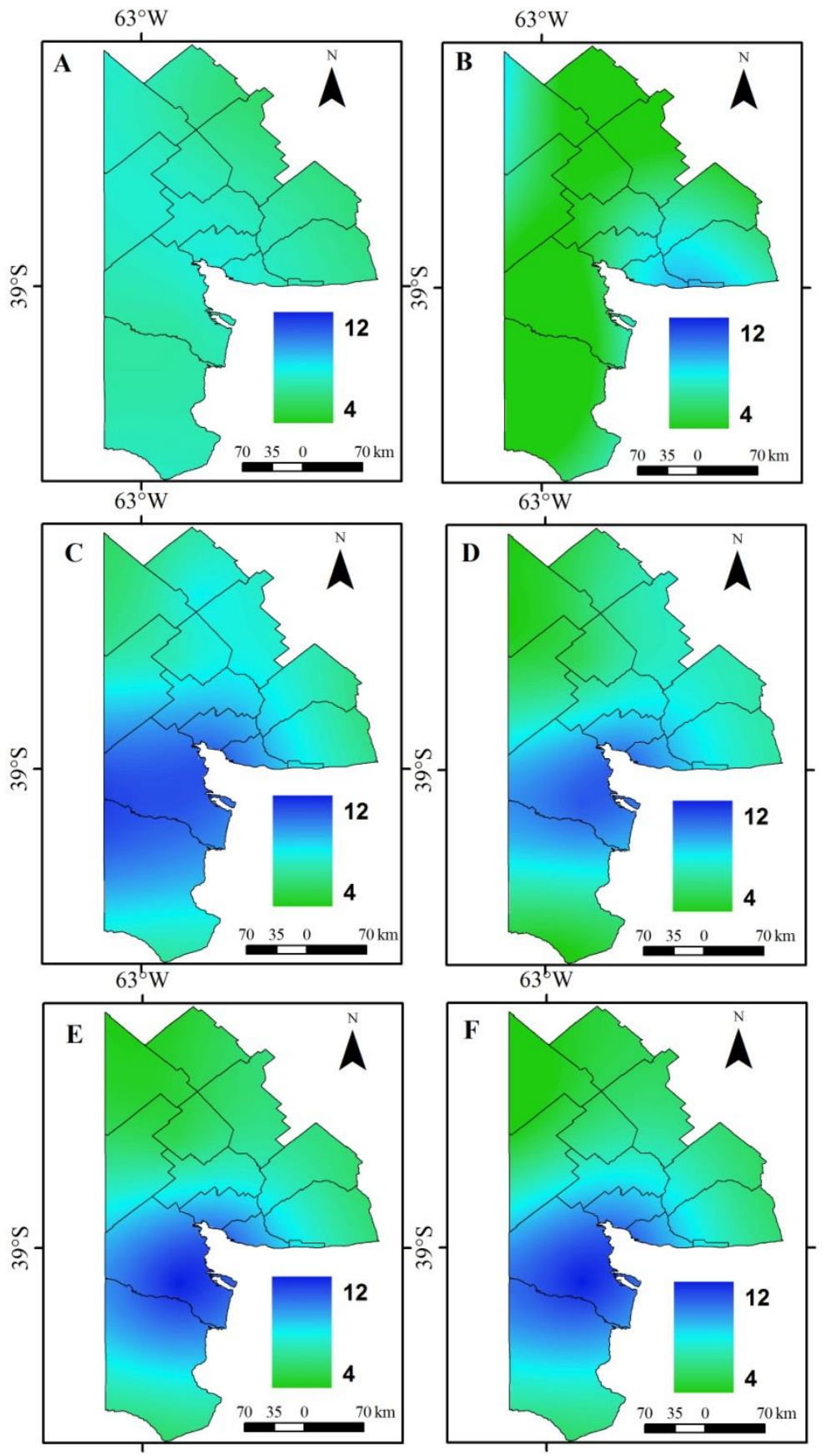

La periodicidad anual de los eventos secos exhibió diferencias espaciales, principalmente hacia el futuro. Entre el pasado y el presente los eventos fueron más frecuentes en el este (3 y 2 años, respectivamente) y se incrementaron hacia el norte y el sur (5,7 y 5,6 años, respectivamente) (Figura 5). En el futuro cercano, se evidenció una situación similar, con eventos menos frecuentes en el norte bajo el escenario RCP 8.5 (6,3 años). Finalmente, en el futuro lejano RCP 4.5, se visualizó un aumento en la frecuencia de los eventos secos en toda el área (3,6 años los menos frecuentes y 2 años los más frecuentes). En el futuro lejano RCP 8.5, toda la región mostró una distribución espacial distinta al resto de los períodos, marcando un área que se extendió de norte 
a sur con eventos secos con mayor frecuencia (1,7 años). Los mismos disminuyeron hacia el este y el oeste (5,3 años) (Figura 5).

FIGURA 5

Distribución espacial de la periodicidad (años) de los eventos secos en A. Pasado reciente, B. Presente, C. Futuro cercano RCP 4.5, D. Futuro cercano RCP 8.5, E. Futuro lejano RCP 4.5 y F.

Futuro lejano RCP 8.5
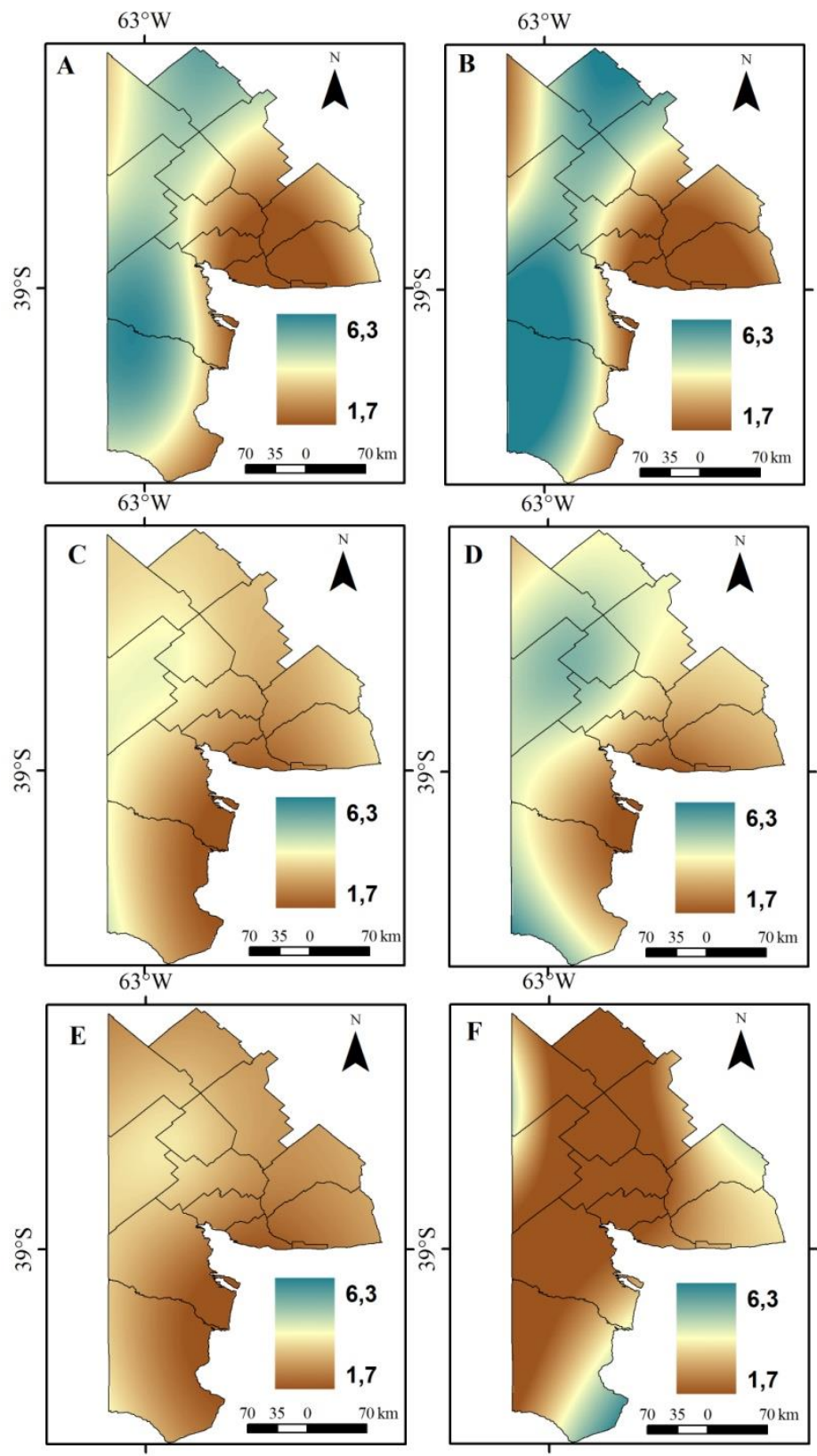

\subsection{EVENTOS HÚMEDOS}

La intensidad de los eventos húmedos se caracterizó por presentar en todos los subperíodos analizados eventos moderadamente húmedos (IEP $>1<1,5$ ) y muy húmedos (IEP $>1,5<2$ ). En el futuro cercano bajo ambos RCP, se identificaron los eventos más intensos en el oeste y el norte del área de estudio (IEP= 1,7 y 1,6, respectivamente) y los menores en el sur (IEP = 1,3 y 1,2, respectivamente) (Figura 6). En el futuro lejano RCP 8.5, se identificaron variaciones considerables, principalmente en el sur que registró los períodos más intensos (IEP $=1,9)$ (Figura $6)$. 
FIGURA 6

Distribución espacial de la intensidad de los eventos húmedos en A. Pasado reciente, B.

Presente, C. Futuro cercano RCP 4.5, D. Futuro cercano RCP 8.5, E. Futuro lejano RCP 4.5 y F.

Futuro lejano RCP 8.5
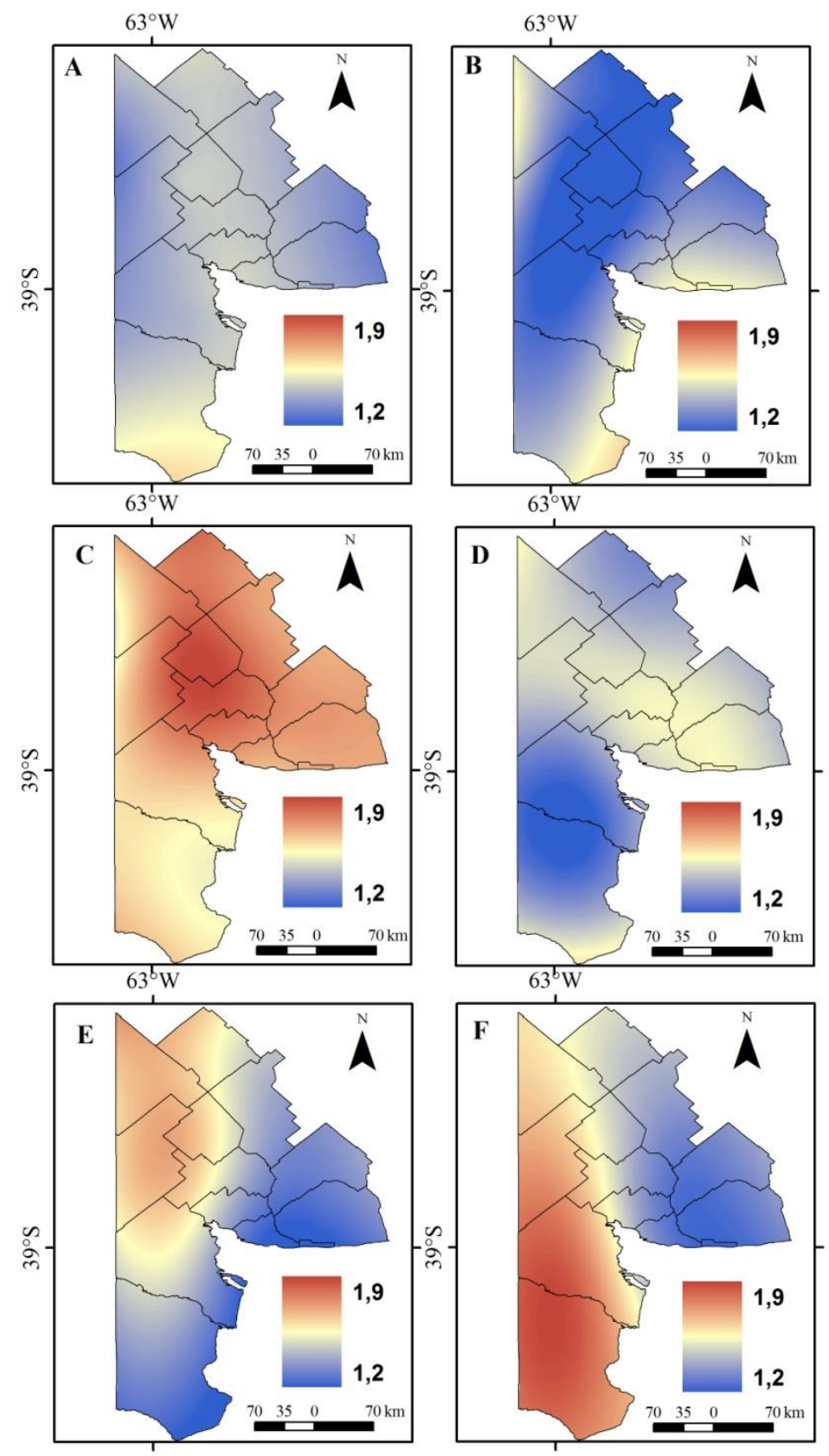

Se evidenció una marcada diferencia entre los períodos pasado reciente y presente. En el primero, los eventos de mayor duración se localizaron en el norte (12 meses) y disminuyeron hacia el sur (5 meses). En el segundo, los períodos húmedos de mayor duración ocurrieron en el sur (14 meses) y disminuyeron hacia el norte (3 meses) (Figura 7). Por otro lado, el futuro cercano, bajo ambos escenarios de RCP, presentó situaciones similares. Los eventos de mayor duración se registraron en el sur y norte del área de estudio ( 17 y 24 meses para el RCP 4.5 y RCP 8.5, respectivamente), y los menores en el centro del área, con una duración de 3 y 5 meses, respectivamente (Figura 7). El futuro lejano, se caracterizó por registrar los eventos más extensos en el norte ( 9 y 13 meses para RCP 4.5 y RCP 8.5, respectivamente), mientras que los más cortos se localizaron en el centro del área de estudio (con 4 meses de duración) (Figura 7). 
FIGURA 7

Distribución espacial de la duración (meses) de los eventos húmedos en A. Pasado reciente, B. Presente, C. Futuro cercano RCP 4.5, D. Futuro cercano RCP 8.5, E. Futuro lejano RCP 4.5 y F. Futuro lejano RCP 8.5
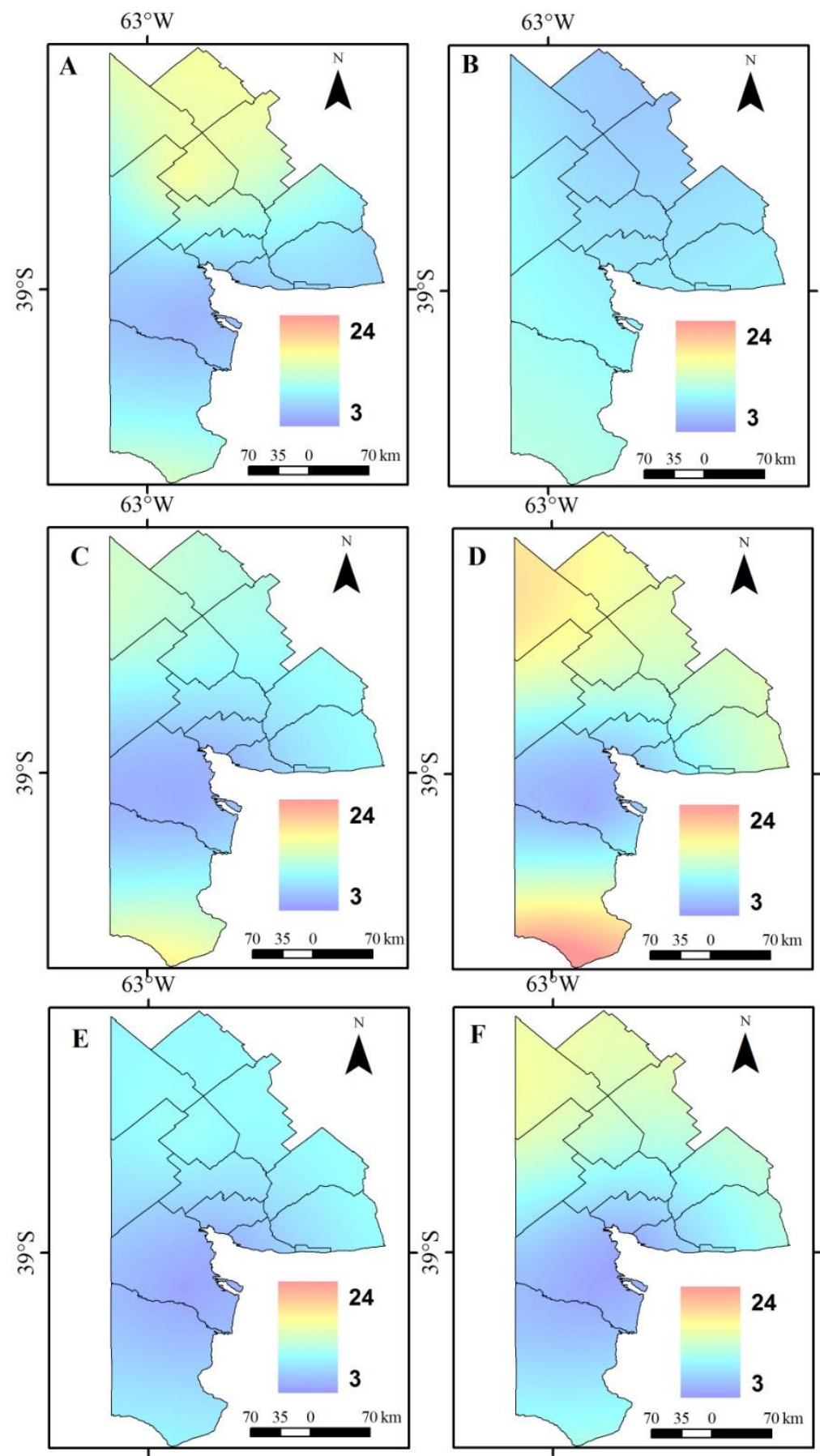

La frecuencia de los eventos húmedos en los distintos períodos mostró variaciones significativas en su distribución espacial. En el pasado reciente, se encontró un mayor número de eventos húmedos en el centro del área de estudio, con un total de 11 y fue menor en el norte y el sur. En el presente, se registraron 11 eventos húmedos en el oeste y disminuyeron a 4 hacia el este (Figura 8). El futuro cercano, en ambos escenarios RCP, tuvo un comportamiento espacial similar, concentrando el mayor número de eventos en el centro del área de estudio (11 en total) y los menores en el norte y el sur. Similar distribución espacial se encontró en el futuro lejano en ambos 
escenarios RCP, pero en ellos la frecuencia de eventos fue mayor (13 para el RCP 4.5 y 14 para el RCP 8.5) (Figura 8).

\section{FIGURA 8}

Distribución espacial de la frecuencia de los eventos húmedos en A. Pasado reciente, B.

Presente, C. Futuro cercano RCP 4.5, D. Futuro cercano RCP 8.5, E. Futuro lejano RCP 4.5 y F.

Futuro lejano RCP 8.5
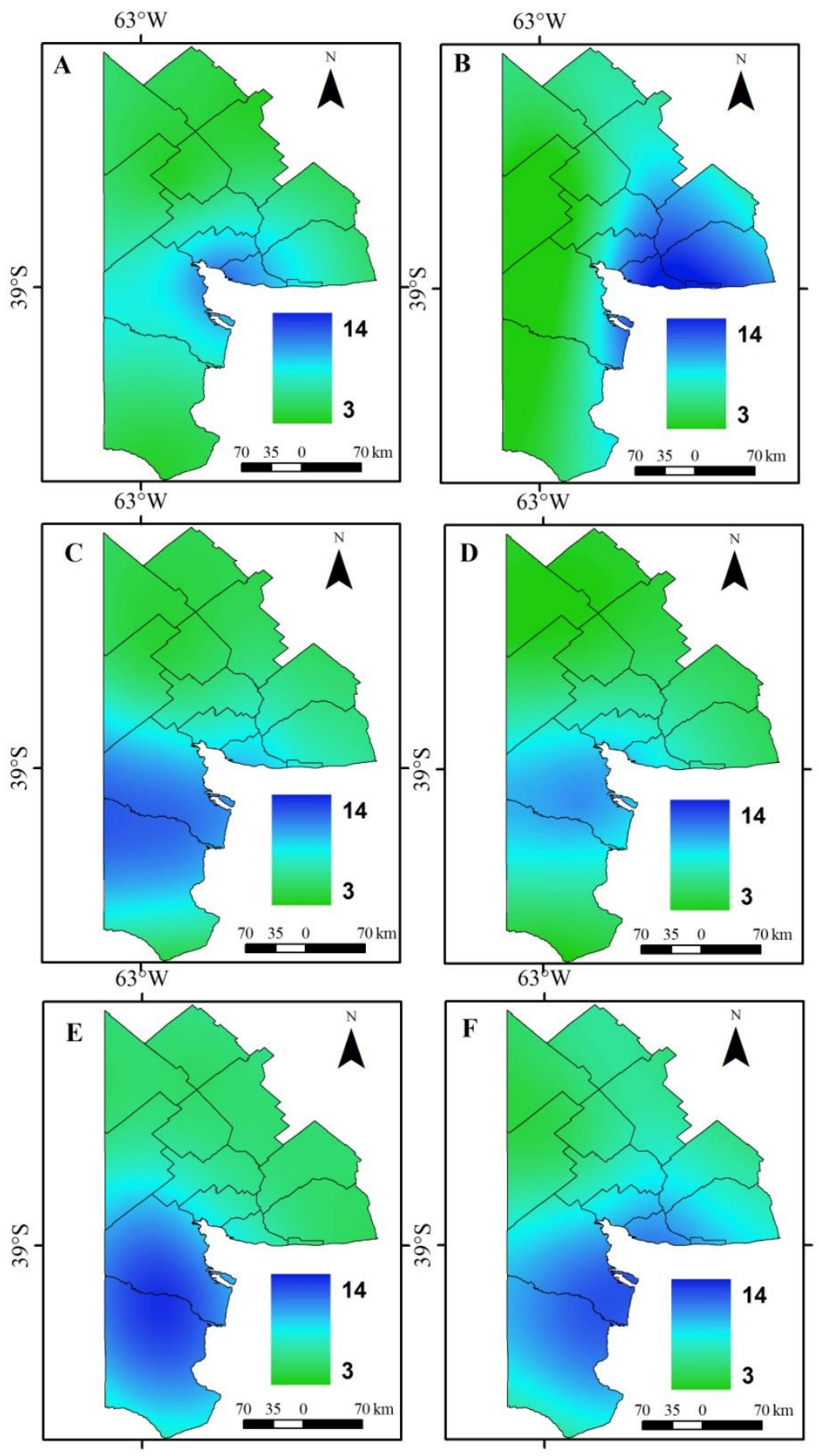

Los eventos húmedos tuvieron mayor periodicidad en el centro del área de estudio (1,4 años) y disminuyeron hacia el oeste y el sur (3,2 años) durante el pasado reciente. El presente, tuvo una menor periodicidad en el noreste, este y sur (6 años) y mayor en el centro y oeste (2,2 años) (Figura 9). El futuro cercano RCP 4.5 presentó una periodicidad de 1,6 años en el sur y centro del 
área de estudio y de 4,6 años hacia el norte. Bajo el RCP 8.5, la distribución espacial de los mismos fue similar, pero con una frecuencia de 1,9 años en el sur y centro y 6 años en el resto de los períodos estudiados (Figura 9). En cambio, el futuro lejano RCP 4.5 tuvo una situación opuesta, dado que los eventos con menor frecuencia ocurrieron en el sur ( 3,5 años) y los de mayor en el norte (2,8 años). Finalmente, el escenario RCP 8.5, se destacó el noroeste del área de estudio dado que fue el que presentó la mayor periodicidad (5,5 años) (Figura 9).

FIGURA 9

Distribución espacial de la periodicidad (años) de los eventos húmedos en A. Pasado reciente, B. Presente, C. Futuro cercano RCP 4.5, D. Futuro cercano RCP 8.5, E. Futuro lejano RCP 4.5 y F. Futuro lejano RCP 8.5
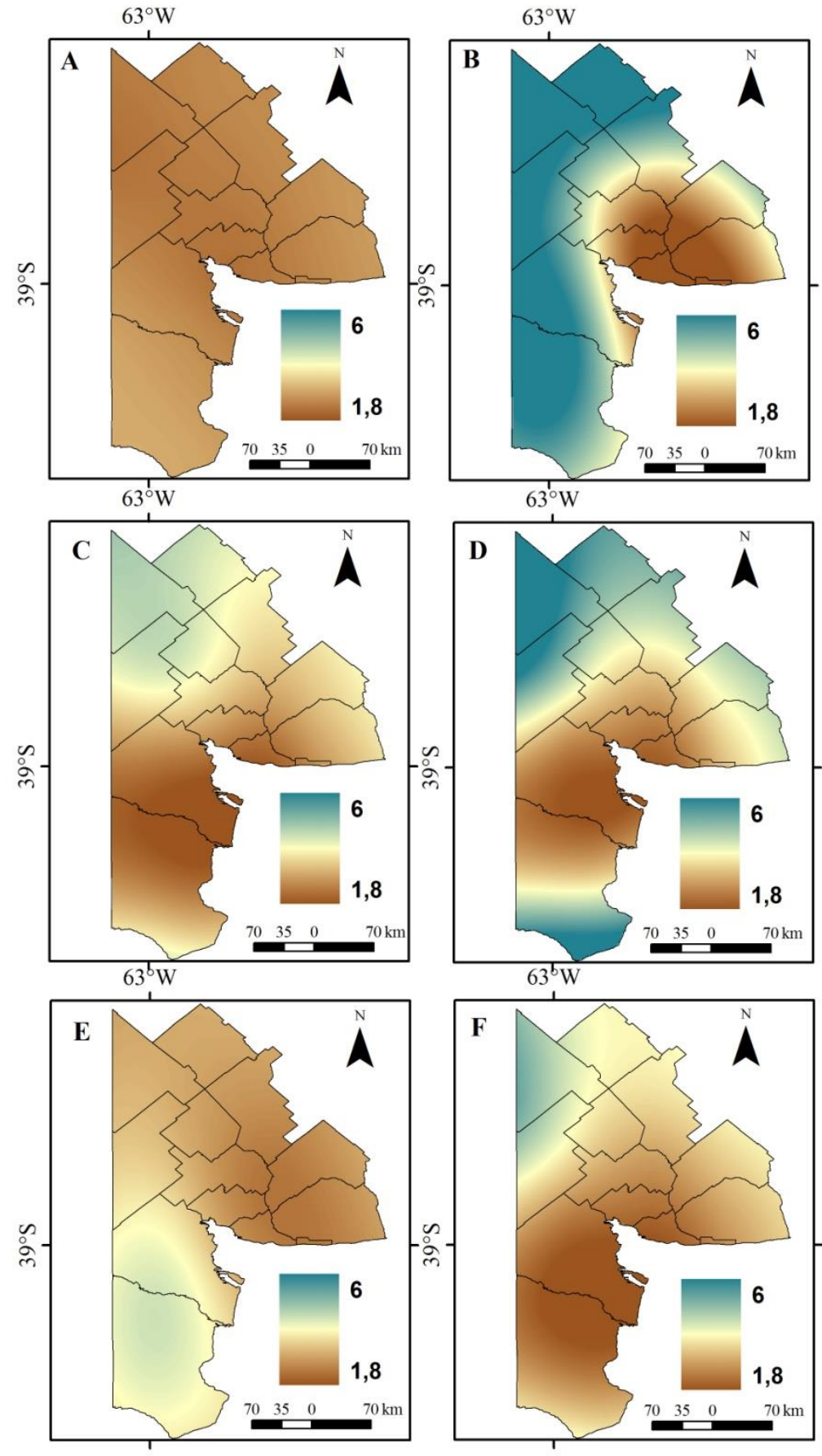

\section{DISCUSIÓN}


El sur de la Región Pampeana es un área caracterizada por una gran heterogeneidad espacial de las precipitaciones (FERRELLI et al., 2019). Se observó que los eventos secos fueron más intensos en al pasado. Esta situación concuerda con lo reportado en el quinto informe del Grupo Intergubernamental de Expertos sobre el Cambio Climático, quienes mencionan que la intensidad de las sequías fue mayor en gran parte del mundo desde 1970 (IPCC, 2013). En este sentido, DAI et al. (2011) concluyeron que durante las últimas tres décadas, todos los continentes presentaron sequías en años simultáneos. Por ejemplo, en América del Sur, ocurrieron durante 1962, 1965/1966, 1971/1972, 1988/1989, 1995/1996 y 2008/2009 (RIVERA y PENALBA, 2014).

Según PENALBA y RIVERA (2013), las sequías a corto y largo plazo en Argentina serán más frecuentes en el siglo XXI y de mayor severidad bajo los dos escenarios RCP. Dicha condición podría generar situaciones críticas para el desarrollo de la agricultura. Por ejemplo, sobre la producción de soja, dado que el déficit hídrico prolongado dificulta el acceso al agua desde la zona radicular, generando pérdidas de rendimiento. Estos efectos podrían ser adversos sobre la población y el ambiente, dado que los períodos secos intensos afectan la capacidad de infiltración, la disponibilidad de nutrientes, el suministro de servicios ecosistémicos y el acceso al agua potable (YAN et al., 2014). Los mismos, al presentar una gran variación espacio-temporal dan cuenta de que el área estudiada tiene una marcada heterogeneidad de regímenes pluviométricos (BEKELE et al., 2017).

Por otro lado, se identificó que los eventos secos tienden a hacerse cada vez más duraderos, sobre todo en el sur del área estudiada ante un escenario RCP 4.5. En el extremo sur de la Región Pampeana, los períodos secos tendrán nuevamente una duración superior a 12 meses en el futuro lejano. Esto podría afectar al período de floración y desarrollo de los cultivos que se emplazan en esa región y hasta se podría necesitar de la implantación de nuevas especies con la finalidad de mantener la actividad agropecuaria (GRASSINI et al., 2011; FERNÁNDEZ-LONG et al., 2013; WORKU et al., 2018).

La extensión de los períodos de sequía, se relaciona directamente con una profundización de las condiciones de aridez. Esto generaría la intensificación de los efectos producidos por la erosión eólica y el aumento de enfermedades sobre la población (REYNOLDS et al., 2005; VICENTE SERRANO et al., 2012; GABELLA et al., 2016). Además, los eventos secos de larga duración impactan sobre las aguas superficiales y subterráneas, pudiendo afectar no sólo a los habitantes, sino también a algunas actividades productivas como la ganadería (ALIAGA et al., 2016; FERRELLI, 2016; BRENDEL et al., 2017).

Por otro lado, se encontró un mayor número de eventos secos? hacia el futuro. Estos resultados concuerdan con lo hallado por PENALBA y RIVERA (2014), quienes encontraron un aumento significativo en el número de eventos de sequía en Argentina durante diferentes sub-períodos del siglo XXI. Esta situación genera la intensificación de los procesos de erosión eólica. Particularmente, este proceso podría afectar al sur del área estudiada, dado que es la que tiene la mayor frecuencia de eventos secos. Por lo tanto, esta región estará expuesta a condiciones de aridez que se incrementarán hacia el futuro lejano (FERRELLI et al., 2019). En el contexto del cambio climático, caracterizado por la ocurrencia de eventos secos y húmedos más extremos, el aumento de las sequías podría impactar negativamente sobre la población. Por ejemplo, como consecuencia de su crecimiento paulatino, la demanda de agua potable aumentarán, pero la generación de períodos recurrentes de sequía podría causar situaciones de imposibilidad para abastecer la demanda (SMITH y KATZ; 2013).

La periodicidad de los eventos tanto secos como húmedos, podría impactar no sólo en la calidad de vida de las personas, sino que también en sus actividades económicas. El sur de la Región Pampeana es una de las áreas productoras de trigo más importantes de Argentina. Los cultivos se desarrollan bajo condiciones de secano por lo que su rendimiento depende directamente de las precipitaciones. Por ejemplo, durante 2008-2009, se generó la sequía más intensa de los últimos veinte años y generó la pérdida de la mayor parte de los cultivos (FERRELLI, 2016). En contraposición, en el período 2001-2002, el evento extremadamente húmedo favoreció el crecimiento de los cultivos, registrándose uno de los mayores rendimientos del periodo 2000-2017 (BRENDEL et al., 2017). 
En el sur del área de estudio, durante el futuro lejano y, bajo condiciones de RCP 8.5, los eventos húmedos serán los más intensos. Estos resultados concuerdan con lo hallado por diversos autores a escala global, quienes afirman que a medida que aumenta la concentración de gases de efecto invernadero, la precipitación será mayor como consecuencia del calentamiento global dado que se generará mayor concentración de vapor de agua en la atmósfera (CHEN et al., 2012; JIANG y FU, 2012; WANG et al., 2012). Por otro lado, los estudios realizados a escala mundial demuestran que las precipitaciones torrenciales y muy fuertes tienen una tendencia positiva, por lo que es esperable que en el futuro, el área de estudio presente precipitaciones concentradas en menor cantidad de días. Esta situación podría intensificar los procesos de erosión hídrica (FERRELLI et al., 2019).

Teniendo en cuenta que el área de estudio presenta un relieve escasamente ondulado con predominancia de un drenaje endorreico, el aumento de la duración de los eventos húmedos podría generar un mayor riesgo de inundaciones (VÁZQUEZ et al., 2003). Además, el caudal de los ríos que drenan sobre esta área responden rápidamente a la intensidad de las precipitaciones (CARBONE et al., 2006). Por lo tanto, el aumento de los eventos húmedos podrían provocar severos daños económicos y humanos, producto del aumento del caudal de los cursos de agua (VOLONTÉ y GIL, 2019).

Por otro lado, es importante destacar que el fenómeno ENSO (El Niño Oscilación Sur, por sus siglas en inglés) es uno de los factores clave que influyen en la variabilidad interanual de las precipitaciones en el sur de América del Sur (PENALBA y RIVERA, 2016). Este fenómeno presentará una mayor variabilidad en el futuro y determinará, por ejemplo, un aumento del riesgo de inundación de numerosas cuencas hidrográficas del mundo (SUN et al., 2020).

Los resultados obtenidos permiten identificar que el análisis de los eventos secos y húmedos pasados, presentes y aquellos derivados de escenarios futuros corresponden una herramienta crucial para el estudio de la variabilidad pluviométrica dentro del contexto del cambio climático (NAM et al., 2015). Se destaca la importancia de los estudios destinados a evaluar las condiciones pluviométricas futuras, dado que permiten reducir el riesgo ambiental al generar información anticipada para la toma de medidas destinadas a mejorar la calidad de vida de la población y el ambiente (WILHITE et al., 2014). Esta información permitirá que los tomadores de decisiones del sur de la Región Pampeana adopten planes de mitigación ante los diversos escenarios futuros a los que se expone el área de estudio con la finalidad de reducir los impactos negativos de estos eventos sobre la población y sus actividades económicas (NAM et al., 2015; FERRELLI et al., 2019).

\section{CONCLUSIONES}

El análisis espacio-temporal de los eventos secos y húmedos, considerando períodos pasados, presentes y futuros en dos escenarios RCP distintos permitió analizar las condiciones a las que estará expuesto el sur de la Región Pampeana dentro de un contexto de cambio climático. Esta adquiere importancia dado que es una de las áreas agrícolas más importantes del país. En ella, la variabilidad pluviométrica futura podría generar grandes impactos negativos sobre las actividades económicas. Por ejemplo, sobre la producción forrajera y de esta manera perjudicar las actividades ganaderas, como así también impactar en la disponibilidad del agua necesaria para el desarrollo de los cultivos. Se encontró que tanto los eventos secos y como los húmedos tienden a ser más intensos y periódicos en la zona sur, por lo que se estima que esta área estará expuesta a una mayor variación de las precipitaciones. Por otro lado, la zona norte y oeste del área de estudio permanecerá con situaciones similares en el futuro, por lo que no estarán sujetas a grandes cambios pluviométricos que pudiesen afectar sus economías locales. La información generada en esta investigación es fundamental para enmarcar planes de manejo del territorio orientados a preservar el ambiente y las actividades económicas, favoreciendo la sustentabilidad ambiental.

\section{AGRADECIMIENTOS}

Papeles de Geografía, 66 (2020), 27-46 
Los autores agradecen al Servicio Meteorológico Nacional (SMN, Argentina), al Instituto Nacional de Tecnología Agropecuaria (INTA, Argentina) y al Centro de Investigaciones del Mar y la Atmósfera de Argentina (CIMA, Argentina) por el suministro de datos analizados para realizar este trabajo. Además, se agradece al Consejo Nacional de Investigaciones Científicas y Técnicas (CONICET) y a la Universidad Nacional del Sur (UNS) por el financiamiento de este estudio.

\section{REFERENCIAS}

AALTO, J., PIRINEN, P., HEIKKINEN, J., y VENÄLÄINEN, A. (2013): "Spatial interpolation of monthly climate data for Finland: comparing the performance of kriging and generalized additive models". Theoretical and applied climatology, vol. 112, $\mathrm{n}^{\circ}$ (1-2), p. 99-111.

ALIAGA, V.S.; FERRELLI, F.; ALBERDI-ALGARAÑAZ, E.D.; BOHN, V.Y. y PICCOLO, M.C. (2016): "Distribution and variability of precipitation in the Pampas, Argentina". Cuadernos de Investigación Geográfica, vol. 42, no 1, p. 261-280.

ALIAGA, V.S.; FERRELLI, F. y PICCOLO, M.C. (2017): "Regionalization of climate over the Argentine Pampas". International Journal of Climatology, vol. 37, p. 1237-1247 Al-Qinna M.I.; Hammouri, N.A.; Obeidat M.M. \& Ahmad F.Y. 2011. Drought analysis in Jordan under current and future climates. Climate. Change, vol. 106, n 3, p. 421-440.

BAIMOUNG, S.; OKI, T.; ARCHEVARAHUPROK, B.; YUTTAPHAN, A. y PANGPOM, M. (2014): Bias correction techniques for meteorological data of A2 scenario climate model output in Chao Phraya River Basin of Thailand. Hydrological Research Letters, vol. 8, $\mathrm{n}^{\circ} 1$, p. 71-76.

BEKELE, F., MOSISA, N., y TEREFE, D. (2017): "Analysis of current rainfall variability and trends over Bale-Zone, South Eastern highland of Ethiopia". Climate Change, vol. 3, no 12, p. 889-902.

BRENDEL, A.S.; BOHN, V.Y. y PICCOLO, M.C. (2017): "Variabilidad de la precipitación y su relación con los rendimientos agrícolas en una región semiárida de la llanura pampeana (Argentina)". Estudios Geográficos, nº 78, p. 7-29.

CARBONE, M. E., PÉREZ, D. E., PICCOLO, M. C., y PERILlO, G. M. E. (2006): "Comportamiento hidrológico del arroyo Claromeco, Argentina". Investigaciones geográficas, $\mathrm{n}^{\circ} 39$, p. 179-193.

CHEN, H.P.J; SUN, J.Q. y CHEN, X.L. (2012): "The projection and uncertainty analysis of summer precipitation in China and the variations of associated atmospheric circulation field". Climatic and Environmental Research, vol. 17, $\mathrm{n}^{\circ}$ 12, p. 171-183.

CHEN, H.P.J.; SUN, J.Q. y CHEN, X.L. (2013): "Future changes of drought and flood events in China under a global warming scenario". Atmospheric and Oceanic Science Letters, vol. 6, $\mathrm{n}^{\circ}$ 1, p.8-13.

COLLAZO, S., BARRUCAND, M., y RUSTICUCCI, M. (2019): "Summer seasonal predictability of warm days in Argentina: statistical model approach". Theoretical and Applied Climatology, vol. 138, no 3-4, p. 1853-1876.

DAI, A. (2011): "Drought under global warming: a review". Wiley Interdisciplinary Reviews: Climate Change, vol. 2, $\mathrm{n}^{\circ}$ 1, p.: 45-65.

DUBROVSKÝ, M., HAYES, M., DUCE, P., TRNKA, M., SVOBODA, M., y ZARA, P. (2014): "Multi-GCM projections of future drought and climate variability indicators for the Mediterranean region". Regional Environmental Change, vol. 14, nº 5, p. 1907-1919.

DU, J., FANG, J., XU, W., y SHI, P. (2013): "Analysis of dry/wet conditions using the standardized precipitation index and its potential usefulness for drought/flood monitoring in Hunan Province, China". Stochastic environmental research and risk assessment, vol. 27, $\mathrm{n}^{\circ}$ 2, p. 377-387.

EDWARDS, C.D.C.; MCKEE, T.B.; DOESKEN, N.J. y KLEIST, J. (1997): "Historical analysis of drought in the United States". En: 77th Conference on climate variations, 77th AMS Annual Meeting, p. 2-7. 
FERNÁNDEZ-LONG, M.E.; MÜLLER, G.V.; BELTRÁN-PRZEKURAT, A. y SCARPATI, O.E. (2013): "Long-term and recent changes in temperature-based agroclimatic indices in Argentina". International Journal of Climatology, vol. 33, $\mathrm{n}^{\circ}$ 7, p. 1673-1686.

FERRELLI, F. (2016): "Efectos de eventos El Niño y La Niña sobre las lagunas del sur de la Región Pampeana (Argentina)". InterEspaço: Revista de Geografia e Interdisciplinaridade, vol. $2, n^{\circ} 6$, p. $122-142$.

FERRELLI, F. (2017): "Variabilidad pluviométrica y sus efectos sobre las coberturas del suelo al sur de la provincia de Buenos Aires, Argentina". Revista Geográfica Venezolana, vol. 58, nº 1, p. 26-37.

FERRELLI, F.; BRENDEL, A.S.; ALIAGA, V.S.; PICCOLO, M.C. y PERILLO, G.M.E. (2019): "Climate regionalization and trends based on daily temperature and precipitation extremes in the south of the Pampas (Argentina)". Cuadernos de Investigación Geográfica, vol. 45, nº 1 , p. 393-416.

GABELLA, J.I. y CAMPO, A. M. (2016): "Procesos de ocupación y construcción del espacio rural pampeano: el caso del partido de Patagones". Huellas, vol. 20, p. 79-99.

GRASSINI, P.; THORBURN, J.; BURR, C. y CASSMAN, K.G. (2011): "High-yield irrigated maize in the Western U.S. Corn Belt: I. On-farm yield, yield potential, and impact of agronomic practices". Field Crops Research, vol. 120, p. 142-150.

HAYES, M. J., WILHELMI, O. V., y KNUTSON, C. L. (2004): "Reducing drought risk: bridging theory and practice". Natural Hazards Review, vol. 5, n 2, p. 106-113.

HAYES, M.; SVOBODA, M.; WALL, N. y WIDHALM, M. (2011): "The Lincoln declaration on drought indices: universal meteorological drought index recommended". Bulletin of the American Meteorological Society, vol. 92, $\mathrm{n}^{\circ}$ 4, p. 485-488.

HEINRICH, G. y GOBIET, A. (2012): "The future of dry and wet spells in Europe: a comprehensive study based on the ENSEMBLES regional climate models". International Journal of Climatology, vol. 32, n 13, p. 1951-1970.

HOUGHTON, R.A. (1993): "Is carbon accumulating in the northern temperate zone?".Global Biogeochem Cycles, vol. 7, p.611-700.

INTERGOVERNMENTAL PANEL ON CLIMATE CHANGE (2013): Climate change 2013: the physical science basis. In Contribution of Working Group I to the Fifth Assessment Report of the Intergovernmental Panel on Climate Change. En: STOCKER, T.F, QIN, D., PLATTNER, G-K., TIGNOR, M., ALLEN, S.K., BOSCHUNG, J., NAUELS, A., XIA, Y., BEX, V. y MIDGLEY, P.M., (Eds), Cambridge University Press, Cambridge, Reino Unido, y New York, Estados Unidos, 203 pp.

Jiang, D.B. \& Fu, Y.H. 2012. Climate change over China with a $2^{\circ} \mathrm{C}$ global warming. Chinese Journal of Atmospheric Sciences, 36(2): 234-246.

KEYANTASH, J., y DRACUP, J. A. (2002): "The quantification of drought: an evaluation of drought indices". Bulletin of the American Meteorological Society, vol. 83, nº 8, p. 1167-1180.

MAENZA, R. A., AGOSTA, E. A., \& BETTOLLI, M. L. (2017): Climate change and precipitation variability over the western 'Pampas' in Argentina. International Journal of Climatology, vol. 37, p. 445-463.

MBAYE, M.L.; HAENSLER, A.; HAGEMANN, S.; GAYE, A.T.; MOSELEY, C. \& AFOUDA, A. (2016): "Impact of statistical bias correction on the projected climate change signals of the regional climate model REMO over the Senegal River Basin". International Journal of Climatology, vol. 36, $\mathrm{n}^{\circ}$ 4, p. 2035-2049.

MCKEE, T. B.; DOESKEN, N.J. y KLEIST, J. (1993): "The relationship of drought frequency and duration to time scales". En: Proceedings the 8th conference on applied climatology, vol. $17, \mathrm{n}^{\circ} 22$, p. $179-183$.

MENAFOGLIO, A.; SECCHI, P. y DALLA, R.M. (2013): "A Universal Kriging predictor for spatially dependent functional data of a Hilbert Space". Electronic Journal of Statistics, vol. 7, p. 2209-2240.

MUKHERJEE, S., MISHRA, A., y TRENBERTH, K. E. (2018): "Climate change and drought: a perspective on drought indices". Current Climate Change Reports, vol. 4, no 2, p. 145-163. 
NAM, W. H., HAYES, M. J., SVOBODA, M. D., TADESSE, T., y WILHITE, D. A. (2015): "Drought hazard assessment in the context of climate change for South Korea". Agricultural Water Management, vol. 160, p. 106-117.

OLLERO OJEDA, A.; BALLARÍN FERRER, D. y MORA MUR, D. (2006): Cambios en el cauce y el llano de inundación del río Ebro (Aragón) en los últimos 80 años. Geographicalia, vol. 50, p. 87-109.

ORLOWSKY, B. y SENEVIRATNE, S.I. (2013): "Elusive drought: uncertainty in observed trends and short-andlong-term CMIP5 projections". Hydrology and Earth System Sciences, vol. $17, \mathrm{n}^{\circ}$ 5, p. 1765-1781.

OTKIN, J. A., ANDERSON, M. C., HAIN, C., y SVOBODA, M. (2015): "Using temporal changes in drought indices to generate probabilistic drought intensification forecasts". Journal of Hydrometeorology, vol. 16, $\mathrm{n}^{\circ}$ 1, p. 88-105.

PALMER, T. N., y RÄISÄNEN, J. (2002): "Quantifying the risk of extreme seasonal precipitation events in a changing climate". Nature, vol. 415, $\mathrm{n}^{\circ}$ 6871, p. 512-514.

PENALBA, O.C. y RIVERA, J.A. (2016): "Regional aspects of future precipitation and meteorological drought characteristics over Southern South America projected by a CMIP5 multi-model ensemble". Journal of Climatology, vol.36, n 2, p. 974-986.

PENALBA, O.C. y RIVERA, J.A (2013): "Future changes in drought characteristics over southern South America projected by a CMIP5 multi-model ensemble". American Journal of Climate Change, vol. 2, $\mathrm{n}^{\circ} 3$, p. 173-182.

REYNOLDS, J.F.; MAESTRE, F.T.; HUBER-SANNWALD, E.; HERRICK, J. y KEMP, P.R. (2005): "Aspectos socioeconómicos y biofísicos de la desertificación". Revista Ecosistemas, vol. 14, no 3 , p. 4-21.

RIVERA J.A. y PENALBA O.C. (2014): "Trends and spatial patterns of drought affected area in Southern South America". Climate, vol. 2, p. 264-278.

ROLLA, A. L., NUÑEZ, M. N., RAMAYÓN, J. J., Y RAMAYÓN, M. E. (2019): "Impacts of climate change on bovine livestock production in Argentina". Climatic Change, vol. 153, $\mathrm{n}^{\circ}$ 3, p. 439-455.

ROLLA, A. L., NUÑEZ, M. N., GUEVARA, E. R., MEIRA, S. G., RODRIGUEZ, G. R., y DE ZÁRATE, M. I. O. (2018): "Climate impacts on crop yields in Central Argentina. Adaptation strategies". Agricultural Systems, vol. 160, p. 44-59.

SHAHID, S., y BEHRAWAN, H. (2008): "Drought risk assessment in the western part of Bangladesh". Natural hazards, vol. 46, n 3, p. 391-413.

SHEFFIELD, J., WOOD, E. F., y RODERICK, M. L. (2012): "Little change in global drought over the past 60 years". Nature, vol. 491, no 7424, p. 435-438.

SMITH, A. B., y KATZ, R. W. (2013): "US billion-dollar weather and climate disasters: data sources, trends, accuracy and biases". Natural hazards, vol. 67, nº 2, p. 387-410.

SOBRAL, B. S., DE OLIVEIRA-JÚNIOR, J. F., DE GOIS, G., PEREIRA-JÚNIOR, E. R., DE BODAS TERASSI, P. M., MUNIZ-JÚNIOR, J. G. R., y ZERI, M. (2019): "Drought characterization for the state of Rio de Janeiro based on the annual SPI index: trends, statistical tests and its relation with ENSO". Atmospheric research, vol. 220, p. 141-154.

SPINONI, J., NAUMANN, G., CARRAO, H., BARBOSA, P., y VOGT, J. (2014): "World drought frequency, duration, and severity for 1951-2010". International Journal of Climatology, vol. 34, no 8, p. 2792-2804.

STRZEPEK, K.; YOHE. G.; NEUMANN, J. y BOEHLERT B. (2010): "Characterizing changes in drought risk for the United States from climate change". Environmental Research Letters, vol. $5, \mathrm{n}^{\circ} 4$, p. 044012.

SUN, Q., MIAO, C., AGHAKOUCHAK, A., MALLAKPOUR, I., JI, D., y DUAN, Q. (2020): "Possible Increased frequency of ENSO-related dry and wet conditions over some major watersheds in a warming climate". Bulletin of the American Meteorological Society, vol. 15, $\mathrm{n}^{\circ}$ 4, p. 409-426.

TOUMA, D., ASHFAQ, M., NAYAK, M. A., KAO, S. C., y DIFFENBAUGH, N. S. (2015): "A multi-model and multi-index evaluation of drought characteristics in the 21 st century". Journal of Hydrology, vol. 526, p. 196-207. 
TSESMELIS, D. E., KARAVITIS, C. A., OIKONOMOU, P. D., ALEXANDRIS, S., \& KOSMAS, C. (2019): "Assessment of the vulnerability to drought and desertification characteristics using the standardized drought vulnerability index (SDVI) and the environmentally sensitive areas index (ESAI)". Resources, vol. 8, nº 1, p. 6.

UYAN, M., y CAY, T. 2010. "Geostatistical methods for mapping groundwater nitrate concentrations". En: 3rd International Conference on Cartography and GIS, vol. 1520, p. 1-7.

VALIENTE, O. M. (2001): "Sequía: definiciones, tipologías y métodos de cuantificación". Investigaciones geográficas, vol. 26, p. 59-80.

VÁZQUEZ, P., ENTRAIGAS, I., GANDINI, M., y USUNOFF, E. (2003): "Identificación de patrones de anegamiento en la cuenca del arroyo del Azul mediante el uso de imágenes Landsat". Revista de Teledetección, vol. 19, p. 43-50.

VICENTE-SERRANO, S.M.; ZOUBER, A.; LASANTA, T. y PUEYO, Y. (2012): "Dryness is accelerating degradation of vulnerable shrublands in semiarid Mediterranean environments". Ecological Monographs, vol. 82, n 4, p. 407-428.

VOLONTÉ, A., y GIL, V. (2019): "Aportes de la hidrogeomorfología histórica en la determinación de áreas inundables a partir de eventos extremos de crecidas". Huellas, vol. 23, $\mathrm{n}^{\mathrm{o}} 1$, p. 11-26.

WANG, H.J.; SUN, J.Q.; CHEN, H.P.; ZHU, Y.L.; ZHANG, Y.; JIANG, D.B. y YANG, S. (2012): "Extreme climate in China: Facts, simulation and projection". Meteorologische Zeitschrift, vol. 21, no 3, p. 279-304.

WANG, X.L.; CHEN, H.; WU, Y.; FENG, Y. y PU, Q. 2010. New techniques for the detection and adjustment of shifts in daily precipitation data series. Journal of Applied Meteorology and Climatology, vol. 49, no 12, p. 2416-2436.

WANG, Y.; DING, M.; VAN WESSEM, J.M.; SCHLOSSER, E.; ALTNAU, S.; VAN DEN BROEKE, M.R. y SUN, W. (2016): "A comparison of Antarctic Ice Sheet surface mass balance from atmospheric climate models and in situ observations". Journal of Climate, vol. $29, \mathrm{n}^{\circ} 14$, p. $5317-5337$.

WILHITE, D. A., SIVAKUMAR, M. V., y PULWARTY, R. (2014): "Managing drought risk in a changing climate: The role of national drought policy". Weather and Climate Extremes, vol. 3, p. 4-13.

WORKU, G.; TEFERI, E.; BANTIDER, A. y DILE, Y.T. (2018): "Observed changes in extremes of daily rainfall and temperature in Jemma Sub-Basin, Upper Blue Nile Basin, Ethiopia". Theoretical and Applied Climatology, vol. 135 n 3-4, p. 1-16.

WU H., SVOBOD M.D., HAYES M.J., WILHITE D.A., y WEN F. (2007): "Appropriate application of the standardized precipitation index in arid locations and dry seasons". International Journal of Climatology, vol.27, p. 65-79.

YAN, L., CHEN, S., XIA, J., y LUO, Y. (2014): "Precipitation regime shift enhanced the rain pulse effect on soil respiration in a semi-arid steppe". PloS one, vol. 9, no 8, p. e104217.

YUAN W. y ZHOU G. (2004): "Comparison between standardized precipitation index and Zindex in China". Acta Phytoecol Sin, vol. 28, n 4, p. 523-529. 